\title{
PROJECTIONS IN NORMED LINEAR SPACES $\left({ }^{1}\right)$
}

\author{
BY \\ DWIGHT B. GOODNER
}

1. Introduction. The object of this paper is to study normed linear spaces which have what we shall call property $\mathrm{P}_{s}$ and to study in particular those normed linear spaces which have property $\mathrm{P}_{1}$. A normed linear space $X$ has property $\mathrm{P}_{s}, s \geqq 1$, if and only if for every normed linear space $Y$ containing $X$, there exists a projection $T,\|T\| \leqq s$, of $Y$ onto $X$.

The terminology used is for the most part as given in Banach [3](2) and Murray [16]. Thus a Banach space means a complete normed linear space; a linear transformation means a bounded and distributive transformation; and a projection means a linear transformation $T$ with the property that $T^{2}=T$. Our notation is, we believe, for the most part standard and it is chosen so as to be the same as that of our references whenever possible.

In $\$ 2$ we study some of the general properties of spaces which have property $\mathrm{P}_{s}$, and we develop a necessary and sufficient condition for a space to have property $\mathrm{P}_{s}$. Lemma 2.3 is of particular importance and is used throughout the remainder of this paper.

In $\$ 3$ we discuss some problems which led to the formulation of Akilov's theorem $[2$, p. 646] and also give a new proof of the theorem. This theorem gives a sufficient condition for certain normed linear lattices to have property $\mathrm{P}_{8}$.

$\$ 4$ is a study of some of the conditions imposed upon the unit sphere of a space which has property $\mathrm{P}_{1}$. An important technique, used in many later proofs, is developed and used to discover several necessary conditions for a space to have property $\mathrm{P}_{1}$.

In $\$ 5$ we characterize normed linear spaces which have property $P_{1}$ and which have extreme points on their unit spheres. A partial ordering is introduced into each space, and then a theorem of Clarkson's is applied to show that the space is equivalent to the space of all continuous real-valued functions over some compact Hausdorff space $H$.

Kakutani $[10$, p. 998] has shown that every abstract $(M)$-space has a concrete representation. In $\$ 6$ we find that an abstract $(M)$-space which has property $\mathrm{P}_{1}$ has a concrete representation as the space of all real-valued continuous functions over some compact Hausdorff space $H$. The space $H$ is found to have the curious property of being extremally disconnected. A

Presented to the Society, September 1, 1949; received by the editors August 23, 1949.

(1) This paper is a condensation of a doctoral dissertation written at the University of Illinois under the direction of Professor Mahlon M. Day.

(2) Numbers in brackets refer to the references cited at the end of the paper. 
necessary and sufficient condition for spaces having property $P_{1}$ to be reflexive is given. Finally, the known examples of spaces having property $\mathrm{P}_{1}$ are discussed.

2. Spaces which have property $P_{8}$. In this section we shall study some of the properties of normed linear spaces which have what we shall call property $\mathrm{P}_{8}$.

2.1 Definition. A normed linear space $X$ has property $\mathrm{P}_{s}, s \geqq 1$, if and only if for every normed linear space $Y$ containing $X$ there exists a projection $T,\|T\| \leqq s$, of $Y$ onto $X$.

In parts of our work it will be necessary to specify that $Y$ is a Banach space. This requirement involves no real loss of generality, since any normed linear space $Y \supset X$ is contained in a complete space $Y^{\prime}$ such that a projection $T$ of norm $s$ of $Y^{\prime}$ onto $X$ is a projection of norm $s$ of $Y$ onto $X$.

Our first theorem shows that a normed linear space which has property $\mathrm{P}$. is a Banach space.

2.2 Theorem. If $X$ is a normed linear space which has property $\mathrm{P}_{s}$, then $X$ is complete.

Proof. Let $Y$ be a Banach space containing $X$. Since $X$ has property $\mathrm{P}_{s}$, there exists a projection $T,\|T\| \leqq s$, of $Y$ onto $X$. Let $\left\{x_{n}\right\}$ be any Cauchy sequence in $X$ and let $\lim _{n \rightarrow \infty} x_{n}=y$. Then $y$ is in $Y$ since $Y$ is complete. Therefore $\lim _{n \rightarrow \infty} T\left(x_{n}\right)=T(y)$ since $T$ is continuous. But $\lim _{n \rightarrow \infty} T\left(x_{n}\right)$ $=\lim _{n \rightarrow \infty} x_{n}$ and hence $T(y)=y$. Therefore $y$ is in $X$ and $X$ is complete.

Two normed linear spaces are equivalent $[3$, p. 180] if there exists between them a one-to-one transformation $U$ which is linear in both directions and $\|U\|=\left\|U^{-1}\right\|=1$. This definition leads us to the following extremely useful lemma.

2.3 Lemma. If a normed linear space $X$ is equivalent to a normed linear space $Y$ and if $X$ has property $\mathrm{P}_{8}$, then $Y$ has property $\mathrm{P}_{8}$.

Proof. Let $W$ be a normed linear space containing $Y$ and let

$$
Z=X \cup(W \cap C Y)=X \cup W^{\prime}
$$

where $C Y$ is the complement of $Y$ and $W^{\prime}=W \cap C Y$. Then if $V$ is the equivalence between $X$ and $Y$, we can extend $V$ to $U$ on $Z$ by defining

$$
U(z)=U\left(w^{\prime}\right)=w^{\prime} \quad \text { if } \quad z \in W^{\prime} \subset Z
$$

and

$$
U(z)=U(x)=V(x)=y \quad \text { if } \quad z \in X \subset Z .
$$

Then $U$ is a one-to-one transformation between $Z$ and $W$.

We define the operation of addition between the elements of $Z$ to mean 


$$
z_{1}+z_{2}=x \quad \text { if } \quad U\left(z_{1}\right)+U\left(z_{2}\right)=w_{1}+w_{2}=y=U(x)
$$

and to mean

$$
z_{1}+z_{2}=w^{\prime} \quad \text { if } U\left(z_{1}\right)+U\left(z_{2}\right)=w_{1}+w_{2}=w^{\prime}=U\left(w^{\prime}\right) .
$$

If $a$ is a real number, we define scalar multiplication by

$$
a z_{1}=z_{2}=U^{-1}\left[a U\left(z_{1}\right)\right] .
$$

Finally, we define a norm in $Z$ by

$$
\|z\|=\|U(z)\|=\|w\| \text {. }
$$

It is easily verified that these definitions make $Z$ a normed linear space and that $U$ is an equivalence between $Z$ and $W$.

Since $X$ has property $\mathrm{P}_{s}$, there exists a projection $T,\|T\| \leqq s$, of $Z$ onto $X$. Let $T^{\prime}=V T U^{-1}$. $T^{\prime}$ is a projection of $W$ onto $Y$ and $\left\|T^{\prime}\right\| \leqq s$. Hence $Y$ has property $\mathrm{P}_{s}$, which was to be shown.

The proof of our next lemma uses the concept of the adjoint transformation $T^{*}$ of a linear transformation $T$. If $X$ is a linear subspace of the normed linear space $Y$ and $T$ is a projection of $Y$ onto $X$, the adjoint mapping $T^{*}$ of $T$ is defined by the relation $T^{*}(f)=g$ implies $g(y)=f(T y)$ for all $y$ in $Y$, where $g \in Y^{*}$ and $f \in X^{*} .\|T *\|=\|T\| ; T^{*}$ is one-to-one on $X^{*}$ into $Y^{*}$; and if $X$ and $Y$ are complete, $X$ is isomorphic to $Y / T(0)^{-1}$ and $T^{*}$ is an isomorphism of $X^{*}$ and $\left[T(0)^{-1}\right]^{\perp}[6$, Theorem 1.13, chap. 4].

2.4 Lemma. If the normed linear space $X$ has property $\mathrm{P}_{8}$ and if $Y$ is a normed linear space containing $X$, there is a subspace $G$ of $Y^{*}$ which is isomorphic to $X^{*}$, and there is a projection $T^{\Delta},\left\|T^{\Delta}\right\| \leqq s$, of $Y^{*}$ onto $G$.

Proof. Since $X$ has property $\mathrm{P}_{s}$ and $Y$ is a normed linear space containing $X$, there is a projection $T,\|T\| \leqq s$, of $Y$ onto $X$. Let $T^{*}$ be the adjoint mapping of $T$ and let $G=T^{*}\left(X^{*}\right)$. Then

$$
G=\left\{h \mid h(y)=h(T y)=T^{*} f(y), h \in Y^{*}, f \in X^{*}\right\} .
$$

Define the transformation $T^{\Delta}$ by

$$
T^{\Delta} h=g \text { implies } g(y)=T^{\Delta} h(y)=h(T y), \quad g \in G .
$$

The reader may verify that $T^{\Delta}$ is a projection, $\left\|T^{\Delta}\right\|=\|T\|$, of $Y^{*}$ onto $G=T^{*}\left(X^{*}\right)$.

The final theorem in this chapter gives a necessary and sufficient condition for a space to have property $\mathrm{P}_{\mathbf{8}}$.

2.5 Theorem. A normed linear space $X$ has property $\mathrm{P}_{\mathrm{s}}$ if and only if for each Banach space $Y$ containing $X$ there exist a closed linear subspace $G$ of $Y^{*}$ and a projection $T^{\lrcorner}$of $Y^{*}$ onto $G$ such that
a. $\left\|T^{\Delta}\right\| \leqq s$, 
b. $Y=G_{\perp} \oplus X$,

c. $T^{\Delta} h=g$ implies $T^{\Delta} h\left(g_{\perp}+x\right)=g\left(g_{\perp}+x\right)=h(x), h \in Y^{*}, g \in G, g_{\perp} \in G_{\perp}$, and $x \in X$.

Proof. Suppose $T^{\Delta}$ and $G$ exist. Then $Y=G_{\perp} \oplus X$ by hypothesis. ( $G_{\perp}$ $=\{y \mid g(y)=0, y \in Y, g \in G\}$.) If we define $T_{1}$ by the relation $T_{1}(y)=T_{1}\left(g_{\perp}+x\right)$ $=x, T_{1}$ is a projection of $Y$ onto $X[16, \mathrm{p} .139]$.

$$
\begin{aligned}
T_{1}^{*}\left(X^{*}\right) & =\left\{h \mid h(y)=T_{1}^{*} f(y)=f\left(T_{1} y\right), f \in X^{*}\right\} \\
& =\left\{h \mid h(y)=h\left(g_{\perp}+x\right)=h(x)\right\}=G .
\end{aligned}
$$

Define $T_{1}^{\Delta}$ by $T_{1}^{\Delta}(h)=g$ implies $g(y)=T_{1}^{\Delta} h(y)=h\left(T_{1} y\right)$ for all $y$ in $Y$. Then $T_{1}^{\Delta}(h)=T^{\Delta}(h)$ for all $h$, so $\left\|T_{1}^{\Delta}\right\|=\left\|T^{\Delta}\right\| \leqq s$. But $\left\|T_{1}\right\|=\left\|T_{1}^{\Delta}\right\| \leqq s$. Therefore, $X$ has property $\mathrm{P}_{s}$.

If $X$ has property $\mathrm{P}_{s}$ and if $Y$ is a Banach space containing $X$, there is a projection $T,\|T\| \leqq s$, of $Y$ onto $X . T^{*}\left(X^{*}\right)=\left[T(0)^{-1}\right]^{\perp}$, and by Lemma 2.4 there is a projection $T^{\Delta},\left\|T^{\Delta}\right\| \leqq s$, of $Y^{*}$ onto $\left[T(0)^{-1}\right]^{\perp}$. Let $G=\left[T(0)^{-1}\right]^{\perp}$. Then $G_{\perp}=\left[T(0)^{-1}\right]_{\perp}^{\perp}=T(0)^{-1}$. Hence $Y=G_{\perp} \oplus X$ since $Y=T(0)^{-1} \oplus X$. Condition $\mathrm{c}$ is satisfied by our definition of $T^{\Delta}$; therefore the proof is complete.

3. Akilov's theorem. Akilov in a short paper [2, p. 643] considered two problems on the extension of linear operators from one normed linear space to another and in a later paper $[1$, p. 417] showed that the properties studied in the two problems are equivalent. Day [5, p. 241] noted that both problems are equivalent to the study of spaces with property $\mathrm{P}_{s}$. We shall state Akilov's problems together with a third problem and show that the three properties involved are equivalent; that is, a space which is a solution (has the property described in the problem) to one of the problems is a solution to the other two. However, before we state the problems a definition is necessary.

3.1 Definition. Given a linear subspace $X$ of a normed linear space $Z$ and a linear transformation $T$ on $X$ into a normed linear space $Y$, we shall call a linear transformation $T_{1}$ on $Z$ into $Y$ an extension of $T$ if $T_{1}(x)=T(x)$ for all $x$ in $X$ and $\left\|T_{1}\right\|=\|T\|$.

Problem 1. Given a normed linear space $Y$, does there exist for each normed linear space $X$, each linear transformation $T$ of $X$ into $Y$, and each normed linear space $Z$ containing $X$, an extension $T_{1}$ of $T$ on $Z$ into $Y$ ?

The familiar Hahn-Banach theorem $[3$, p. 55] provides an affirmative answer to problem 1 if the normed linear space $Y$ is the real line.

Problem 2. Given a normed linear space $X$, does there exist for each normed linear space $Y$, each linear transformation $T$ from $X$ into $Y$, and each normed linear space $Z$ containing $X$, an extension $T_{1}$ of $T$ on $Z$ into $Y$ ?

Problem 3. Given a normed linear space $X$, does there exist for every normed linear space $Z$ containing $X$, an extension $T_{1}$ of the identity transformation $I$ of $X$ onto $X$ such that $T_{1}$ transforms $Z$ onto $X$ ?

In this case $T_{1}$, if it exists, is a projection of norm one of $Z$ onto $X$ and 
any space $X$ which provides a solution to problem 3 has property $\mathrm{P}_{1}$.

Proof that problem 2 implies problem 3. Suppose $X$ is a space which satisfies the conditions of problem 2 and let $Y=X$. Then the identity transformation $I$ of $X$ onto $X$ is a linear transformation of $X$ into $Y$. Hence, by problem 2 there is an extension $T_{1}$ of $I$ such that $T_{1}$ transforms $Z$ into $X$; that is, there is a projection $T_{1},\left\|T_{1}\right\|=1$, of $Z$ onto $X$.

Proof that problem 1 implies problem 3. Suppose $Y$ is a space which satisfies the conditions of problem 1 and let $X=Y$. Then the identity transformation $I$ of $X$ onto $X$ is a linear transformation of $X$ into $Y$. Hence by problem 1 there exists an extension $T_{1}$ of $I$ such that $T_{1}$ transforms $Z$ into $X$; that is, there is a projection $T_{1},\left\|T_{1}\right\|=1$, of $Z$ onto $X$.

Proof that problem 3 implies problem 2. Suppose $X$ is a space which satisfies the conditions of problem 3 and let $T$ be a linear transformation of $X$ into $Y$. If $Z$ contains $X$, there is a projection $P,\|P\|=1$, of $Z$ onto $X$. Then the linear transformation $T_{1}=T P$ is an extension of $T$, and $T_{1}$ transforms $Z$ into $Y$.

Proof that problem 3 implies problem 1. Suppose $Y$ is a normed linear space which satisfies the condition of problem 3 and let $T$ be a linear transformation of a normed linear space $X$ into $Y$. If $m(S)$ is the space of all bounded real-valued functions on $S$, the unit sphere of $Y^{*}$, we can imbed $Y$ in $m(S)$ by an equivalence $U$ defined by $U(y)=y^{\prime}$ implies $s(y)=y^{\prime}(s)$ for all $s$ in $S$. Suppose $Z$ is a normed linear space containing $X$. Then by Phillips' Corollary $7.2[18$, p. 538] the linear transformation $U T$ of $X$ into $m(S)$ has an extension $T_{1}$ which transforms $Z$ into $m(S)$. Since $Y$ has property $\mathrm{P}_{1}$, $Y^{\prime}=U(Y)$ has property $\mathrm{P}_{1}$ by Lemma 2.3 . Hence there is a projection $P,\|P\|$ $=1$, of $m(S)$ onto $Y^{\prime}$. Let $T^{\prime}=U^{-1} P T_{1} . T^{\prime}$ is the required extension of $T$ which transforms $Z$ into $Y$.

The proof of our next theorem is similar to the proof that problem 3 implies problem 1.

3.2 Theorem. If the normed linear space $Y$ has property $\mathrm{P}_{s}$, if $T$ is a projection of norm $t$, and if $T$ projects $Y$ onto a subspace $X$ of $Y$, then $X$ has property $\mathrm{P}_{s t}$.

Proof. If $m(S)$ is the space of all bounded real-valued functions on $S$, the unit sphere of $Y^{*}$, we can imbed $Y$ in $m(S)$ by an equivalence $U$ defined by $U(y)=y^{\prime}$ implies $s(y)=y^{\prime}(s)$ for all $s$ in $S$. Suppose $Z$ is a normed linear space containing $X$. Then by Phillips' Corollary $7.2[18$, p. 538] the linear transformation $U$ of $X$ into $m(S)$ has an extension $V$ which transforms $Z$ into $m(S)$. Since $Y$ has property $\mathrm{P}_{s}, Y^{\prime}=U(Y)$ has property $\mathrm{P}_{s}$ by Lemma 2.3. Hence there is a projection $P,\|P\| \leqq s$, of $m(S)$ onto $Y^{\prime}$. Let $T^{\prime}=T U^{-1} P V$. Then $T^{\prime}$ is a projection of norm less than or equal to st, and $T^{\prime}$ projects $Z$ onto $X$. Hence $X$ has property $\mathrm{P}_{s t}$.

Akilov's study of problems 1 and 2 led him to the formulation of a suffi- 
cient condition (which we shall call Akilov's theorem) for a normed linear space to have property $\mathrm{P}_{8}$. His proof of the condition was based on Kantorovitch's extension theorem [13, p. 285]. The proof we present here resembles the proof of the Hahn-Banach theorem and does not make use of the Kantorovitch theorem.

A space is of type $B_{1}^{+}\left[2\right.$, p. 643] if it is simultaneously of types $K_{5}$ and $B_{1}$ [11, pp. 210-212]. Using this definition we state and prove Akilov's theorem $[2$, p. 646] in the following form [5, p. 241].

3.3 THEOREM. If the normed linear space $X$ is of type $B_{1}^{+}$, if the unit sphere $C$ of $X$ has a least upper bound $x_{0}$, and if $\left\|x_{0}\right\|=s$, then $X$ has property $\mathrm{P}_{8}$.

Proof. Let $Z$ be a normed linear space containing $X$, let $p$ be a sub-additive, positive-homogeneous transformation on $Z$ into $X$, and let $f$ be a distributive transformation on $X$ into $X$ such that $f(x) \leqq p(x)$ for all $x$ in $X$. Substituting $X$ for the real numbers in a proof of Banach's [3, p. 28], we can show there exists a maximal function $F_{0}$ which is defined on all of $Z$ and which has the properties that $F_{0}(x)=f(x)$ for all $x$ in $X$ and $F_{0}(z) \leqq p(z)$ for $z$ in $Z$.

Let $p$ be the sub-additive positive-homogeneous function defined by the relation $p(z)=x_{0}\|z\|$. If we take the identity function $I$ of $X$ onto $X$ as $f$, $I(x) \leqq p(x)$ for all $x$, and $I$ can be extended to a distributive function $T$ such that $T(z) \leqq p(z)=x_{0}\|z\|$ and $T(x)=x$. $T$ is a projection, $\|T\|=s$, of $Z$ onto $X$. Hence $X$ has property $P_{s}$ and the proof is complete.

Let $l_{p, n}$ be the space of ordered $n$-tuples of real numbers $\{a\}$ $=\left\{\left(a_{1}, a_{2}, \cdots, a_{n}\right)\right\}, 1 \leqq n<\infty$, with the norm $\|a\|=\left(\sum_{i=1}^{n}\left|a_{i}\right|^{p}\right)^{1 / p}, 1 \leqq p$ $<\infty$. If the usual partial ordering-that is, $a^{\prime} \geqq a^{\prime \prime}$ if and only if $a_{i}^{\prime} \geqq a_{i}^{\prime \prime}$ for all $i$-is introduced, it can be easily verified that $l_{p, n}$ satisfies the conditions of Akilov's theorem and has property $\mathrm{P}_{s}, s=n^{1 / p}$.

In a later section we will consider other examples of spaces which satisfy Akilov's theorem. However, for the present, we shall content ourselves with a brief examination of two spaces which do not have property $\mathrm{P}_{s}$ but which satisfy all but one of the conditions of the theorem.

Sobczyk $[19$, p. 945$]$ proved there is no projection of $(m)$, the space of bounded sequences, onto its subspace $\left(c_{0}\right)$, the space of sequences convergent to zero. Hence $\left(c_{0}\right)$ does not have property $\mathrm{P}_{s} .\left(c_{0}\right)$ is a space of type $B_{1}^{+}$but its unit sphere has no upper bound in the space.

Fichtenholtz and Kantorovitch [7, p. 92] proved there is no projection of $M(0,1)$, the space of all bounded functions on the unit interval, onto its subspace $C(0,1)$, the space of continuous functions on the unit interval. Hence $C(0,1)$ does not have property $\mathrm{P}_{s .} C(0,1)$ is a space of type $B_{1}$, and its unit sphere has a least upper bound of norm one. Hence $C(0,1)$ satisfies all conditions of Akilov's theorem except the one requiring that all sets bounded above have a least upper bound in the space. 
4. The unit sphere of a space which has property $\mathrm{P}_{1}$. Let $X$ be a normed linear space and let $C$ be the unit sphere of $X$. The purpose of this section is to study some of the conditions which are imposed on $C$ if $X$ has property $\mathrm{P}_{1}$. In order to do this we make frequent use of a technique which is of importance in much of our later work. Briefly it is this: our first lemma tells us that $X$ has property $\mathrm{P}_{1}$ if and only if the unit sphere $K$ of any normed linear space $Y$ containing $X$ projects on to $C$; we let $Y$ be a linear (as yet unnormed) space containing $X$ and of dimension one greater than the dimension of $X$ and find points in $Y$ not in $X$ which are symmetric about the origin and such that their convex hull intersects $X$ in or on $C$; we then norm $Y$ by letting $K$, the unit sphere of $Y$, be the closed convex hull of these points and $C$; then if we have selected the points of $K$ so that at least one of them must project outside of $C$, we know that $X$ does not have property $\mathrm{P}_{1}$.

4.1 Lemma. The normed linear space $X$ with unit sphere $C$ has property $\mathrm{P}_{1}$ if and only if for each normed linear space $Y$ containing $X$ there is projection $T$ of $Y$ onto $X$ which projects the unit sphere $K$ of $Y$ onto $C$.

Proof. If for each $Y$ containing $X$ there exists a projection $T$ of $Y$ onto $X$, such that $T(K)=C$, then $\|T\|=\operatorname{lub}_{\|y\| \leqq 1}\|T(y)\|=\operatorname{lub}_{\|y\| \leqq 1}\|x\|=1$. Hence $X$ has property $\mathrm{P}_{1}$.

Suppose that for some $Y$ containing $X$ there is no projection of $Y$ onto $X$ or that if a projection $T$ of $Y$ onto $X$ exists, there is a $y_{0}$ in $K$ such that $T\left(y_{0}\right)$ is not in $C$. Then

$$
\|T\| \geqq\left\|T\left(y_{0}\right)\right\|>1
$$

and $X$ does not have property $\mathrm{P}_{1}$.

4.2 Theorem. If $p$ and $q \neq p,-p$ are extreme points of the unit sphere $C$ of the normed linear space $X$ which has property $P_{1}$, the line segment connecting $p$ and $q$ lies wholly on the surface of $C$.

Proof. Assume the line segment connecting $p$ and $q$ does not lie wholly on the surface of $C$. Then $(p+q) / 2$ is inside $C$ since $C$ is convex. Also $(p+q) / 2$ is not equal to zero for $q$ is not equal to $-p$. Hence there exists a point $r$ $=t(p+q)$ such that $1 / 2<t \leqq 1$ and $r$ is in or on $C$. Let $b=(2 t-1)(p+q)$. Let $Y$ be a linear (as yet unnormed) space containing $X$ and of dimension one greater than the dimension of $X$; let $y_{0}$ be a fixed element of $Y$ not in $X$; let $K$, the unit sphere in $Y$, be the smallest closed convex set containing $C$ and the eight points $\pm p \pm y_{0}, \pm q \pm\left(y_{0}+b\right)$.

Our next step is to show that $K \cap X=C$. Let $S$ be the convex hull of $\pm p \pm y_{0}, \pm q \pm\left(y_{0}+b\right)$. If a point in $C$ is of the form $a s+(1-a) c, s$ in $S$, $c$ in $C, a \neq 0$ a real number, then $s$ is in $S \cap X$; so it suffices for our purpose to prove $S \cap X$ is contained in $C . S$ intersects $X$ in a polygon which can have extreme points only where a line segment of $S$ connects two extreme points 
of $S$ which are not in $C$. Checking all possibilities will show that the extreme points of $S \cap X$ are $\pm p, \pm q$, and $\pm r$. But these points were chosen so that the hexagon they determine is in $C$. Hence $K \cap X=C$.

Our final step is to show that every projection of $Y$ onto $X$ carries a point of $K$ outside of $C$. Let $T$ be a projection of $Y$ onto $X$ and let $T\left(y_{0}\right)=x_{0}$. Then $T$ carries the line segment from $p+y_{0}$ to $p-y_{0}$ onto the line segment from $p+x_{0}$ to $p-x_{0}$. Since $p$ is an extreme point of $C$, either one end point of the segment is outside of $C$ or both end points equal $p$ and $x_{0}=0$. For $T$ to be of norm one, the latter condition must hold. Then $T\left(q \pm\left(y_{0}+b\right)\right)=q \pm b$. But $q$ is an extreme point so $\|q+b\|>1$ or $\|q-b\|>1$, and consequently $\|T\|>1$. Hence our assumption was false and the theorem is proved.

4.3 Theorem. If the normed linear space $X$ has property $\mathrm{P}_{1}$ and if $p$ is an extreme point of the unit sphere $C$ of $X$, then any point $q$ of norm one, $q \neq p,-p$, can be connected to either $p$ or $-p$ by a line segment lying entirely on the surface of $C$.

Proof. Assume there is a point $q$ of norm one such that the line segments $p q$ and $-p q$ do not lie on the surface of $C$. Then $(p+q) / 2$ and $(-p+q) / 2$ are inside $C$ and are not the zero element. Hence there is a point $r_{1}$ in $C$ between the points $(p+q) / 2$ and $(p+q) /\|p+q\|$ and a point $r_{2}$ in $C$ between the points $(-p+q) / 2$ and $(-p+q) /\|-p+q\|$. Let $e_{1}=1-\left\|r_{1}\right\|$ and $e_{2}=1-\left\|r_{2}\right\|$. $e_{1}>0$ and $e_{2}>0$. Let $e$ equal the smaller of $e_{1}$ and $e_{2}$ and let $k$ equal the larger of $\|p+q\|$ and $\|-p+q\|$. Then if $b_{1}=(e / k)(p+q)$ and $b_{2}=(e / k)(-p+q),\left\|b_{1}\right\|$ $\leqq e$ and $\left\|b_{2}\right\| \leqq e$. Let $Y$ be a linear (as yet unnormed) space which contains $X$ and which is of dimension one greater than the dimension of $X$, and let $y_{0}$ be an element of $Y$ not in $X$. Let $K$, the unit sphere in $Y$, be the smallest convex set containing $C$ and the points $\pm p \pm y_{0}, \pm q \pm\left(y_{0}+b_{1}+b_{2}\right)$. Then $K \cap X=C$.

Since $X$ has property $P_{1}$, there is a projection $T,\|T\|=1$, of $Y$ onto $X$. Let $T\left(y_{0}\right)=x_{0} . T$ carries the line segment from $p+y_{0}$ to $p-y_{0}$ onto the line segment from $p+x_{0}$ to $p-x_{0}$. Since $p$ is an extreme point of $C$, either one end of the line segment is outside of $C$ or both ends equal $p$ and $x_{0}=0$. For $T$ to be of norm one the latter condition must hold. Then $T\left(q+y_{0}+b_{1}+b_{2}\right)$ $=q+b_{1}+b_{2}$. $\left\|q+b_{1}+b_{2}\right\|=|1+2 e / k|>1$. But $\left\|q+y_{0}+b_{1}+b_{2}\right\|=1$; so, by Lemma 4.1, $X$ does not have property $\mathrm{P}_{1}$, and our assumption has led to a contradiction. Hence the theorem is proved.

4.4 Lemma. Let $X$ be a normed linear space with unit sphere $C$ and let $Y$ be a linear space containing $X$ and of dimension one greater than the dimension of $X$. Let $E$ be a subset of $C$ of diameter $d<2$ and let $a=$ minimum $(1,2 / d-1)$. If $y_{0}$ is fixed in $Y$ and is not in $E$, and if $K$ is the closed convex hull on $C$ and

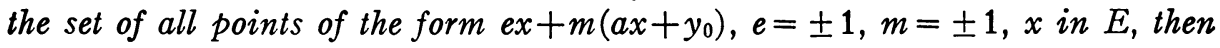
$K \cap X=C$. 
Proof. Let $x_{1}$ and $x_{2}$ be any two points of $E$ and consider the convex hull $C^{\prime}$ on $e x_{1}+m\left(a x_{1}+y_{0}\right)$ and $e^{\prime} x_{2}+m^{\prime}\left(a x_{2}+y_{0}\right)$ where $e, e^{\prime}, m$, and $m^{\prime}$ are equal to \pm 1 and $a$ is positive. $C^{\prime} \cap X \subset C$ if $w=\left(e x_{1}+a x_{1}+e^{\prime} x_{2}-a x_{2}\right) / 2$ is in $C$. If $e=e^{\prime}, w=\left((e+a) x_{1}+(e-a) x_{2}\right) / 2$ and $a \leqq 1$ implies $\|w\| \leqq 1$. If $e=-e^{\prime}$, $w=(e+a)\left(x_{1}-x_{2}\right) / 2$ and this is in $C$ if $|e+a| \leqq 2 /\left\|x_{1}-x_{2}\right\|$. This is possible for $a>0$ only when $\left\|x_{1}-x_{2}\right\|<2$; a suitable choice is, in fact, $2 /\left\|x_{1}-x_{2}\right\|-1$. Then $w$ lies in $C$ if $a=$ minimum $(1,2 / d-1)$. Hence $K \cap X=C$.

4.5 CoRollary. If the normed linear space $X$ has property $\mathrm{P}_{1}$, each extreme point of the unit sphere $C$ is at a distance 2 from every other extreme point of $C$.

Proof. Form $K$ as described in Lemma 4.4 and let $K$ be the unit sphere of $Y$. Since $X$ has property $\mathrm{P}_{1}$, there is a projection $T,\|T\|=1$, of $Y$ on to $X$. Hence $e x_{1}+m\left(a x_{1}+y_{0}\right)$ in $K$ must project into $e x_{1}+m\left(a x_{1}+T\left(y_{0}\right)\right)=e x_{1}$ $+m\left(a x_{1}+u\right)$ in $C$. Then $a x_{1}+u$ is in $\left(C-x_{1}\right) \cap\left(C+x_{1}\right)$. If $x_{1}$ is an extreme point, $\left(C-x_{1}\right) \cap\left(C+x_{1}\right)=0$, so $a x_{1}+u=0$ or $u=-a x_{1}$. If both $x_{1}$ and $x_{2} \neq x_{1}$ are extreme points of $C, u=-a x_{1}=-a x_{2}$, so $a$ must be zero. Then by Lemma 4.4 the set $E$ consisting of $x_{1}$ and $x_{2}$ must be of diameter 2 .

By a face of the unit sphere $C$ of the normed linear space $X$ we shall mean a maximal convex subset of the surface of $C$.

4.6 Lemma. If the normed linear space $X$ has property $\mathrm{P}_{1}$ and if $E$ of diameter less than or equal to one is a subset of the unit sphere $C$, then all faces of $C$ which contain points of $E$ intersect in some point of $C$.

Proof. Let $Y$ be a linear (as yet unnormed) space containing $X$ and of dimension one greater than the dimension of $X$. Form $K$ as described in Lemma 4.4 and let $K$ be the unit sphere of $Y$. There is a projection $T$, $\|T\|=1$, of $Y$ onto $X$ since $X$ has property $\mathrm{P}_{1}$. If $e x_{1}+m\left(a x_{1}+y_{0}\right)$ is in $K$, $e x_{1}+m\left(a x_{1}+T\left(y_{0}\right)\right)$ is in $C$. Then $a x_{1}+T\left(y_{0}\right)=a x_{1}+u$ is in $\left(C-x_{1}\right) \cap\left(C+x_{1}\right)$. Hence $u$ is in $\left(C-x_{1}-a x_{1}\right) \cap\left(C+x_{1}-a x_{1}\right)$. Since the diameter of the set $E$ which contains $x_{1}$ is less than or equal to one, $a=1, u$ is in $\left(C-2 x_{1}\right) \cap C$, and $-u$ is in $C \cap\left(C+2 x_{1}\right)$. If $\left\|x_{1}\right\|=f_{1}\left(x_{1}\right)=\left\|f_{1}\right\|=1$ for $f_{1}$ in $X^{*}$, then $H_{1}$ $=\left\{x \mid f_{1}(x)=1\right\}$ separates $C$ from $C+2 x_{1}$, so $u$ lies in $H_{1} \cap C$. Suppose $x_{1}$ and $x_{2}$ are in $E$ and $\left\|x_{1}\right\|=\left\|x_{2}\right\|=1$; then there exist $f_{1}$ and $f_{2}$ in $X^{*}$ such that $\left\|f_{1}\right\|=\left\|f_{2}\right\|=1$ and $f_{1}\left(x_{1}\right)=f_{2}\left(x_{2}\right)=1$. Let $F_{i}=\left\{x \mid x \in C\right.$ and $\left.f_{i}(x)=1, i=1,2\right\}$. $-u$ is in $C \cap\left(C+2 x_{2}\right)$ as well as $C \cap\left(C+2 x_{1}\right)$. Then $F_{1} \cap F_{2} \supset-u$. Hence all faces of $C$ which contain points of $E$ intersect in a common point.

The proof of our first corollary makes use of Mazur's theorem $[14$, p. 73] that if $K$ is a convex body in a normed linear space $X$ and if $E$ is a flat subset of $X$ which contains no interior points of $K$, then there exists a hyperplane $H$ such that $H \supseteq E$ and $K$ lies on one side of $H$.

4.7 Corollary. If $X, Y, E, y_{0}, T$, and $u$ are as in the lemma, if $\left\|x_{1}\right\|$ $=\left\|x_{2}\right\|=1$, if $x_{1} \in E$, and if the line segment from $x_{1}$ to $x_{2}$ is on the surface of $C_{2}$ then the line segment from $-u$ to $x_{2}$ is on the surface of $C$. 
Proof. By Mazur's theorem there is a hyperplane $H$ containing the line through $x_{1}$ and $x_{2}$ and such that $C$ lies on one side of $H$. Then by the lemma $-u$ belongs to $H \cap C$. Since $H \cap C$ is a convex set on the surface of $C$, the line segment from $-u$ to $x_{2}$ must lie on the surface of $C$.

The next corollary is from Akilov [1, p. 417].

4.8 Corollary. No $n$-dimensional normed linear space $(1<n<\infty)$ such that the tangent hyperplane at each point of the unit sphere is unique has property $\mathrm{P}_{1}$.

Proof. Let $X$ be an $n$-dimensional normed linear space $(1<n<\infty)$ with property $\mathrm{P}_{1}$. Since $X$ is finite-dimensional its unit sphere $C$ has an extreme point $e$. Let $E$ be the set of all points on the surface of $C$ at a distance less than or equal to $1 / 2$ from $e$. Let $Y, T, y_{0}$, and $u$ be as in the lemma. Then all tangent hyperplanes to $C$ which contain points of $E$ must pass through $e$. Hence a tangent hyperplane at $e$ is not unique.

4.9 Theorem. A normed linear space $X$ does not have property $\mathrm{P}_{1}$ if there exists in $X$ a hyperplane $H=\{x \mid f(x)=1=\|f\|\}$ such that $H \cap C$ ( $C$ the unit sphere of $X)$ is an $n$-dimensional simplex, $3 \leqq n$.

Proof. Let $Y$ be a linear space containing $X$ and of dimension one greater than the dimension of $X$. Suppose $H$ exists and let $p_{1}, p_{2}, \cdots, p_{n}$ be the vertices of $F=H \cap C$. The centers of the faces of $F$ are $q_{i}=(1 /(n-1)) \sum_{j \neq i} p_{j}$ where $i, j=1,2, \cdots, n$.

$$
\left\|q_{i}-q_{k}\right\|=\frac{1}{n-1}\left\|p_{k}-p_{i}\right\| \leqq 1
$$

Hence $E=\left\{q_{i}\right\}, i=1,2, \cdots, n$, is a set of diameter less than or equal to one. Let $y_{0}$ be a fixed point in $Y$ which is not in $X$ and construct the points $\pm q_{i} \pm\left(q_{i}+y_{0}\right), i=1,2, \cdots, n$. Then if $K$ is the closed convex hull of these points and $C, K \cap X=C$ by Lemma 4.4. Let $K$ be the unit sphere of $Y$. If $X$ has property $\mathrm{P}_{1}$ there exists a projection $T,\|T\|=1$, of $Y$ onto $X . T\left( \pm q_{i}\right.$ $\left.\pm\left(q_{i}+y_{0}\right)\right)= \pm q_{i} \pm\left(q_{i}+u\right)$ is in $C$ and by our previous work $-u$ must be on every face of $F$. Since this is impossible, $X$ cannot have property $\mathrm{P}_{1}$.

Our next corollary is from Akilov [1, p. 417].

4.10 CoRollaRY. No abstract $(L)$-space $(A L)$ of dimension greater than or equal to 3 has property $\mathrm{P}_{1}$.

Proof. Realize $(A L)$ as a space $L^{\prime}$ of measurable functions which are integrable with respect to a completely additive measure on some space $S\left[9\right.$, p. 533]; take three disjoint subsets $S_{i}, i=1,2,3$, of $S$ such that each $S_{i}$ is of positive finite measure, and let $L$ be the subspace of $L^{\prime}$ spanned by the three characteristic functions of these sets. Then let $T f=g$ mean that $g(s)=0$ if $s$ is not in one of the three sets and let $g(s)$ be equal to 
the average value of $f$ over $S_{i}, i=1,2,3$, if $s$ belongs to $S_{i}$. It may be easily verified that $T$ is a projection, $\|T\|=1$, of $L^{\prime}$ onto $L$. Then if $L^{\prime}$ has property $\mathrm{P}_{1}, L$ has property $\mathrm{P}_{1}$ by Theorem 3.2. But $L$ does not have property $\mathrm{P}_{1}$ by Theorem 4.9. Hence $L^{\prime}$ does not have property $\mathrm{P}_{1}$. Lemma 2.3 then tells us that $(A L)$ does not have property $\mathrm{P}_{1}$.

5. A characterization of certain normed linear spaces which have property $P_{1}$. The purpose of this section is to characterize those normed linear spaces which have property $\mathrm{P}_{1}$ and which have extreme points on their unit spheres. Since an equivalence preserves extreme points, such a characterization includes spaces with property $\mathrm{P}_{1}$, any one of which has an extreme point on its unit sphere; hence it includes finite-dimensional spaces, conjugate spaces, and reflexive spaces, which have property $P_{1}$. The extreme point is used to introduce a partial ordering in $X$. This partial ordering enables us to show that a space with property $P_{1}$ is equivalent to the space of all realvalued continuous functions over some compact Hausdorff space $H$. From this the characterization follows easily.

Let $C$, the unit sphere of the normed linear space $X$ which has property $\mathrm{P}_{1}$, have an extreme point $u$. Let

$$
C_{u}=\left\{x \mid x=t x^{\prime}, x^{\prime} \in C+u, t \text { real, } t \geqq 0\right\} .
$$

$C_{u}$ is closed [4, p. 849]. We define $x_{1} \geqq x_{2}, x_{1}$ and $x_{2}$ in $X$, if and only if $x_{1}-x_{2}$ is in $C_{u}$. This definition gives $X$ a partial ordering such that the unit sphere $C$ of $X$ consists of just those elements $x$ with $-u \leqq x \leqq u[4$, p. 850].

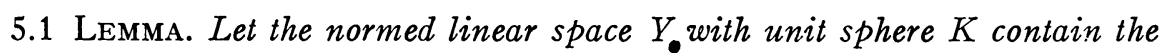
normed linear space $X$ in which the unit sphere $C$ has an extreme point $u$. $A$ projection $T$ of $Y$ onto $X$ maps $K$ onto $C$ if and only if it maps $K_{u}=\left\{y \mid y=t y^{\prime}\right.$, $\left.y^{\prime} \in K+u, t \geqq 0\right\}$ onto $C_{u}$.

Proof. Suppose $T$ maps $K$ onto $C$ and let $y=t y^{\prime}$ be in $K_{u}$ where $t$ is a real number greater than or equal to zero and $y^{\prime}$ is in $K+u$. Then $y=t\left(y^{\prime \prime}+u\right)$ where $y^{\prime \prime}$ is in $K$.

$$
T(y)=T\left(t\left(y^{\prime \prime}+u\right)\right)=t\left(T\left(y^{\prime \prime}\right)+T(u)\right)=t\left(x^{\prime \prime}+u\right)
$$

where $x^{\prime \prime} \in C$. Hence

$$
T(y)=t\left(x^{\prime \prime}+u\right)=t\left(x^{\prime}\right) \in C_{u} \quad \text { where } \quad x^{\prime} \in C+u .
$$

Hence $T$ maps $K_{u}$ onto $C_{u}$.

Suppose $T$ does not map $K$ onto $C$; that is, suppose there is a $y$ in $K$ such that $T(y)=x$ is not in $C$. Then $\|x\|>1$. We assume $x$ is in $C_{u}-u$, for otherwise we are done. Then $x+u \in C_{u}$; that is, $x \geqq-u$. $-y \in K$ since $y \in K$ and hence $T(-y)=-x \notin C$ since $x \notin C$. Suppose $-x+u$ is in $C_{u}$. Then $u \geqq x$. Hence $-u \leqq x \leqq u$ and $x$ is in $C$, a contradiction since $\|x\|>1$. Then $-x+u$ is not in $C_{u}$ as we assumed above. Thus $u-y$ is in $K_{u}$ but $T(u-y)=u-x$ is 
not in $C_{u}$. Hence if $T(K) \neq C, T\left(K_{u}\right) \neq C_{u}$; that is, $T\left(K_{u}\right)=C_{u}$ implies $T(K)$ $=C$.

5.2 LEMMA. If the normed linear space $X$ has property $\mathrm{P}_{1}$, if the unit sphere $C$ of $X$ has an extreme point $u$, and if $x_{1} \geqq x_{2}$ means $x_{1}-x_{2}$ is in $C_{u}$, then every set in $X$ bounded above has a least upper bound.

Proof. Suppose there is a set $A$ in $X$ such that $A$ is bounded above but has no least upper bound. Let $B$ be the set of all upper bounds of $A$ and expand $A$ to include all lower bounds of $B$. Then $a \leqq x \leqq b, a$ in $A$ and $b$ in $B$, is impossible since $x$ would be a least upper bound of the original set $A$. Let $Y$ be a linear (as yet unnormed) space of dimension one greater than the dimension of $X$, let $y_{0}$ be a fixed element of $Y$ not in $X$, and let $K_{u}^{\prime}$ be the smallest half-cone with vertex at the origin containing $C_{u}, y_{0}+B$, and $-y_{0}$ $-A$. Then $K_{u}^{\prime} \cap X=C_{u}$.

If there exists a projection $T$ of $K_{u}^{\prime}$ onto $C_{u}$, for $b$ in $B, T\left(y_{0}+b\right)=T\left(y_{0}\right)$ $+b \in C_{u}$ and $b \geqq-T\left(y_{0}\right)$. Likewise for $a$ in $A, T\left(-y_{0}-a\right)=-T\left(y_{0}\right)-a \in C_{u}$ and $-T\left(y_{0}\right) \geqq a$. Then $-T\left(y_{0}\right)$ would have to lie between $A$ and $B$, an impossibility. Hence there does not exist a projection of $K_{u}^{\prime}$ onto $C_{u}$.

Let $\alpha$ be a real number greater than zero and let $b \in B$. If $x=\alpha b, x+\alpha y_{0}$ $=\alpha\left(b+y_{0}\right) \in K_{u}^{\prime}$. If $x+\alpha y_{0} \in K_{u}^{\prime}, x \in K_{u}^{\prime}-\alpha y_{0}$ and $x=\alpha\left(b+y_{0}\right)-\alpha y_{0}=\alpha b$ for some $b$ in $B$. Hence for $\alpha>0, x+\alpha y_{0} \in K_{u}^{\prime}$ if and only if $x=\alpha b$ with $b$ in $B$. Similarly, for $\alpha<0, x+\alpha y_{0} \in K_{u}^{\prime}$ if and only if $x=\alpha a$ with $a$ in $A$, and for $\alpha=0, x+\alpha y_{0} \in K_{u}^{\prime}$ if and only if $x \in C_{u}$.

We are now ready to show that $K_{u}^{\prime}$ is closed. Suppose $\alpha$ is a real number greater than zero. Let $\alpha_{n}\left(x_{n}+y_{0}\right) \rightarrow x+\alpha y_{0}$ where $\alpha_{n} x_{n}+\alpha_{n} y_{0} \in K_{u}^{\prime} . \alpha_{n}>0$ for $n$ large enough since $\alpha_{n} \rightarrow \alpha$. $\alpha_{n} x_{n} \rightarrow x$ and by a previous result $x_{n} \in B$ for $\alpha_{n}>0$, so for large enough $n, \alpha_{n} x_{n} \geqq \alpha_{n} a$ for each $a$ in $A$. For $\epsilon>0$ there exists an $n(\epsilon)$ such that $(\epsilon / 2) u \geqq\left(\alpha-\alpha_{n}\right) a$ if $n>n(\epsilon)$. Also there exists $m(\epsilon)$ such that if $n>m(\epsilon),(\epsilon / 2) u \geqq \alpha_{n} x_{n}-x$; that is, $x \geqq \alpha_{n} x_{n}-(\epsilon / 2) u$. Combining these results gives $x \geqq \alpha a-\epsilon u$. But since $C_{u}$ is closed, $x \geqq \alpha a$ for all $a$ in $A$. Then $x \in \alpha B$ by the definition of $B$ and $x+\alpha y_{0}=\alpha\left(b+y_{0}\right) \in K_{u}^{\prime}$. By similar arguments we see that the same conclusion holds for $\alpha<0$ and $\alpha=0$. Hence $K_{u}^{\prime}$ is closed.

Let

$$
K=\left\{x+\alpha y_{0}|| \alpha \mid \leqq 1, x+\alpha y_{0}+u \in K_{u}^{\prime}, u-x-\alpha y_{0} \in K_{u}^{\prime}\right\} .
$$

$K$ may be used as the unit sphere of $Y$. Then

$$
K+u=\left\{x+\alpha y_{0}|| \alpha \mid \leqq 1, x+\alpha y_{0} \in K_{u}^{\prime}, 2 u-x-\alpha y_{0} \in K_{u}^{\prime}\right\} .
$$

$K_{u} \subseteq K_{u}^{\prime}$ since $K+u \subset K_{u}^{\prime}$. Let $z \in K_{u}^{\prime}$; then

$$
z=x+\alpha\left(-a-y_{0}\right)+\beta\left(b+y_{0}\right)
$$

where $x \in C_{u}, a \in A, b \in B$, and $\alpha$ and $\beta$ are real numbers greater than or equal to zero. For all real numbers $\lambda>0, \lambda z \in K_{u}^{\prime}$. To get $\lambda|-\alpha+\beta| \leqq 1$ we need only 
take $\lambda$ small. It remains to be shown that for $\lambda$ sufficiently small $2 u-\lambda z \in K_{u}^{\prime}$. In this connection it suffices to show that for $k$ sufficiently large, $k u-z \in K_{u}^{\prime}$, for by setting $\lambda=2 / k>0$, we have $2 u-\lambda z \in \lambda K_{u}^{\prime}=K_{u}^{\prime}$. Take $k_{1}=\max (\|x\|, 1)$. Then $k_{1} u-x \in C_{u}$. Take $k_{2}$ so that $k_{2} u+\alpha a \in \alpha B$, that is, so that $k_{2} u+\alpha a=\alpha b^{\prime}$ for some $b^{\prime}$ in $B$. Take $k_{3}$ so that $k_{3} u-\beta b \in-\beta A$, that is, $k_{3} u-\beta b=-\beta a^{\prime}$ for some $a^{\prime}$ in $A$. Then $k u-z=\left(k_{1}+k_{2}+k_{3}\right) u-x-\alpha\left(-a-y_{0}\right)-\beta\left(b+y_{0}\right)=k_{1} u$ $-x+\alpha\left(y_{0}+b^{\prime}\right)+\beta\left(-y_{0}-a^{\prime}\right) \in K_{u}^{\prime}$. Hence $z \in K_{u}$ and $K_{u} \supseteq K_{u}^{\prime}$. But we have previously shown that $K_{u} \subseteq K_{u}^{\prime}$ so $K_{u}=K_{u}^{\prime}$.

There is a projection $T,\|T\|=1$, of $Y$ onto $X$ since $X$ has property $\mathrm{P}_{1}$. Then $T(K)=C$ by Lemma 4.1 and $T\left(K_{u}\right)=C_{u}$ by Lemma 5.1. But we have shown above that $T\left(K_{u}^{\prime}\right)=T\left(K_{u}\right) \neq C_{u}$. Hence our assumption is false and $A$ must have a least upper bound. This completes the proof of the lemma.

We now quote a theorem of Clarkson's [4, p. 847] with the notation modified slightly so as to be consistent with the notation of our previous work.

5.3 Theorem. Let $X$ be a real Banach space. In order that there shall exist a compact Hausdorff space $H$ such that $X$ is equivalent to $C(H)$, the space of all real-valued continuous functions over $H$, the following two conditions are necessary and sufficient:

A. There exists an element $u$ of $C$, the unit sphere of $X$, such that any element of $S$, the surface of $C$, is co-facial either with $u$ or $-u$.

B. The half-cone $C_{u}$ has the property that the intersection of two of its translates is itself a translate. work.

The following lemma is a direct result of this theorem and our previous

5.4 Lemma. If the normed linear space $X$ has property $\mathrm{P}_{1}$ and if the unit sphere $C$ of $X$ has an extreme point $u$, then $X$ is equivalent to a space $C(H)$ of all real-valued continuous functions on some compact Hausdorff space $H$.

Proof. $X$ satisfies Clarkson's condition A by Theorem 4.3. To show that $X$ satisfies Clarkson's condition B, we let $x_{1}$ and $x_{2}$ be any two elements in $X$ and prove that $C_{u}+x_{1}$ and $C_{u}+x_{2}$ intersect in a translate of $C_{u}$.

$$
C_{u}+x_{1}=\left\{x \mid x-x_{1} \geqq 0\right\} . \quad C_{u}+x_{2}=\left\{x \mid x-x_{2} \geqq 0\right\} .
$$

Hence $\left(C_{u}+x_{1}\right) \cap\left(C_{u}+x_{2}\right)=\left\{x \mid x-x_{1} \geqq 0, x-x_{2} \geqq 0\right\}$. Let $k=\max \quad\left(\left\|x_{1}\right\|\right.$, $\left.\left\|x_{2}\right\|, 1\right) . k u \geqq x_{1}$ and $k u \geqq x_{2}$ since $u \geqq x$ if $\|x\| \leqq 1$. Then $x_{1}$ and $x_{2}$ are bounded above. Therefore by Lemma 5.2 there exists in $X$ an element $x_{3}$ which is the least upper bound of $x_{1}$ and $x_{2}$. Hence $\left(C_{u}+x_{1}\right) \cap\left(C_{u}+x_{2}\right)=C_{u}+x_{3}$ is a translate of $C_{u}$. Then $X$ satisfies Clarkson's conditions and is therefore equivalent to a space $C(H)$ of continuous functions over some compact Hausdorff space $H$.

Lemma 5.4 enables us to give another proof of Corollary 4.5. If the space 
$X$ has property $\mathrm{P}_{1}$ and an extreme point on its unit sphere, $X$ is equivalent to a space $C(H)$ by Lemma 5.4. The extreme points of the unit sphere of $C(H)$ are all at a distance two from each other. Since an equivalence preserves distances and extreme points, the extreme points of the unit sphere of $X$ are at a distance two from each other.

5.5 Theorem. A normed linear space $X$ with an extreme point $u$ on its unit sphere $C$ has property $\mathrm{P}_{1}$ if and only if $X$ is equivalent to a space $Y$ of type $B_{1}^{+}$ whose unit sphere $S$ has a least upper bound $v$ of norm one.

Proof. Suppose $X$ is equivalent to a space $Y$ of type $B_{1}^{+}$whose unit sphere $S$ has a least upper bound $v$ of norm one. Then $Y$ has property $\mathrm{P}_{1}$ by Akilov's theorem. Therefore $X$ has property $\mathrm{P}_{1}$ by Lemma 2.3 .

If $X$ has an extreme point on its unit sphere $C$ and if $X$ has property $\mathrm{P}_{1}$, then $X$ is equivalent to a space $C(H)$ of all real-valued continuous functions over some compact Hausdorff space $H$, by Lemma 5.4. $C(H)$ is a space of type $B_{1}$ when given its usual norm and partial ordering. But $C(H)$ has property $\mathrm{P}_{1}$ and hence is of type $B_{1}^{+}$by Lemma 5.2. Let $v$ in $C(H)$ be the element such that $v(t)=1$ for all $t$ in $H .\|v\|=1$ and $v$ is the least upper bound of $S$. Hence $C(H)$ is a space of type $B_{1}^{+}$whose unit sphere has a least upper bound $v$ of norm one.

\section{Abstract $(M)$-spaces which have property $\mathrm{P}_{1}$.}

6.1 Theorem. An abstract $(M)$-space $X$ which has property $\mathrm{P}_{1}$ has a unit element.

Proof. $X$ is isometric and lattice isomorphic to a space $C\left(H ; t_{\alpha}, t_{\alpha}^{\prime}\right.$; $\left.\lambda_{\alpha} ; \alpha \in \mathfrak{M}\right)$ by Kakutani's theorem [10, p. 998]. Let $Q$ be the transformation between the two spaces. $Q$ preserves property $\mathrm{P}_{1}$ by Lemma 2.3 , so $C\left(H ; t_{\alpha}, t_{\alpha}^{\prime} ; \lambda_{\alpha} ; \alpha \in \mathfrak{M}\right)$ has property $\mathrm{P}_{1}$. Hence there is a projection $T$, $\|T\|=1$, of $C(H)$, the space of all continuous functions on $H$, onto its subspace $C\left(H ; t_{\alpha}, t_{\alpha}^{\prime} ; \lambda_{\alpha} ; \alpha \in \mathfrak{M}\right)$.

We now show that if $y$ is in $C(H)$ and $y \geqq 0$, then $T(y)=x \geqq 0$. If $y=0$, $T(y)=0$ and it is true for this case. Suppose $y>0$ but $T(y)=x$ is not greater than or equal to 0 . Then $\|y\|>0$ and $\|x\|>0$. Let $y_{0}=y /\|y\|$ and $x_{0}=x /\|y\|$. Then $\left\|y_{0}\right\|=1$ and $\left\|x_{0}\right\|>0$. Let $x^{\prime}=x_{0} \wedge 0 . x^{\prime}$ is in $C\left(H ; t_{\alpha}, t_{\alpha}^{\prime} ; \lambda_{\alpha} ; \alpha \in \mathfrak{M}\right)$ since the space is a lattice; $x^{\prime}<0 ;\left\|x^{\prime}\right\|=k>0$. Since $H$ is compact, there is a $t$ in $H$ such that $x^{\prime}(t)=-k$. Let $x^{\prime \prime}=x^{\prime} /\left\|x^{\prime}\right\|=x^{\prime} / k$. $\left\|x^{\prime \prime}\right\|=1, x^{\prime \prime}(t)=-1$, and $x^{\prime \prime}<0$. Then $\left\|y_{0}+x^{\prime \prime}\right\| \leqq 1$ and $T\left(y_{0}+x^{\prime \prime}\right)=x_{0}+x^{\prime \prime}$. But $\left|\left(x_{0}+x^{\prime \prime}\right)(t)\right|$ $=|-k-1|>1$, which is a contradiction since $\|T\|=1$. Therefore our assumption was false and $T(y) \geqq 0$ when $y \geqq 0$.

Let $v$ in $C(H)$ be the element such that $v(s)=1$ for all $s$ in $H$. If $y$ is in $C(H)$ and $\|y\| \leqq 1$, then $v-y \geqq 0$; in particular if $x$ is in $C\left(H ; t_{\alpha}, t_{\alpha}^{\prime} ; \lambda_{\alpha} ; \alpha \in \mathfrak{M}\right)$ and $\|x\| \leqq 1$, then $v-x \geqq 0$. Hence $T(v-x)=e-x \geqq 0$ since $T$ is a positive transformation. Then the element $u=Q^{-1}(e)$ in $X$ is the unit element of $X$. 
6.2 Corollary. An abstract $(M)$-space $X$ which has property $\mathrm{P}_{1}$ has a concrete representation of the form $C(H)$, where $C(H)$ is the space of all realvalued continuous functions over some compact Hausdorff space $H$.

Proof. By Theorem 6.1, $X$ has a concrete representation of the form $C\left(H ; t_{\alpha}, t_{\alpha}^{\prime} ; \lambda_{\alpha} ; \alpha \in \mathfrak{M}\right)$ which has a unit element. Then by a proof of Kakutani's $\left[10\right.$, p. 1005], $C\left(H ; t_{\alpha}, t_{\alpha}^{\prime} ; \lambda_{\alpha} ; \alpha \in \mathfrak{M}\right)$ is actually the entire space $C(H)$.

In Lemma 5.4 and again in Corollary 6.2 we found that certain spaces which have property $P_{1}$ are each equivalent to a space of all continuous realvalued functions over some compact Hausdorff space $H$. We shall now turn our attention to the compact Hausdorff space $H$ and find that it possesses a very curious property known as extremal disconnectivity. A Hausdorff space $H$ is said to be extremally disconnected if every pair of disjoint open subsets of $H$ have disjoint closures.

6.3 TheOREm. The space $C(H)$ of all real-valued continuous functions on a compact Hausdorff space $H$ has property $\mathrm{P}_{1}$ if and only if $H$ is extremally disconnected.

Proof. Suppose $C(H)$ has property $\mathrm{P}_{1}$. Then by Lemma 5.2 every set of elements of $C(H)$ which is bounded above has a least upper bound. Stone has shown $[20$, p. 186] that if $C(H)$ has this property, then every open set in $H$ has a closure which is open. Then by a theorem of Hewitt's [8, p. 326], the closures of disjoint open sets in $H$ are disjoint. From this we conclude that $H$ is extremally disconnected.

$C(H)$ is a space of type $B_{1}$ and its unit sphere has a least upper bound $e$ of norm one where $e$ is the element identically equal to one on $H$. If $H$ is extremally disconnected, every set in $C(H)$ that is bounded above has a least upper bound $\left[20\right.$, p. 186]. Hence $C(H)$ is a space of type $B_{1}^{+}$whose unit sphere has a least upper bound $e$ of norm one. Then by Akilov's theorem, $C(H)$ has property $\mathrm{P}_{1}$.

6.4 THEOREM. The unit sphere $S$ of $C(H)$, the space of all bounded continuous real-valued functions on an extremally disconnected Hausdorff space $H$, is the closed convex hull of its extreme points.

Proof. Let $K$ be the convex hull of the extreme points of $S$; let $F$ be any element of $S$; let $\epsilon$ be a real number greater than zero; let $N$ be the smallest integer greater than $2 / \epsilon$; let the elements of $H$ be denoted by $x$ 's. Our first step is to show that there is an element of $K$ within $\epsilon$ of $F$, that is, there is an element $g$ in $K$ such that $\|F-g\| \leqq \epsilon$. Let

$$
E_{1}=\{x \mid F(x)>1-\epsilon\} .
$$

$E_{1}$ is open $\left[15\right.$, p. 40]. Then $E_{1}^{\prime}=c E_{1}$, the closure of $E_{1}$, is open and closed since $H$ is extremally disconnected $\left[8\right.$, p. 326]. Hence $C E_{1}^{\prime}$, the complement 
of $E_{1}^{\prime}$, is open and closed. The characteristic function $C_{E_{1}^{\prime}}$ of $E_{1}^{\prime}$ is upper semicontinuous since $E_{1}^{\prime}$ is closed and lower semi-continuous since $E_{1}^{\prime}$ is open. Hence $C_{E_{1}^{\prime}}$ is continuous. Let

$$
E_{2}=\{x \mid F(x)>1-2 \epsilon\} .
$$

Since $E_{2}$ is open, $c E_{2}$ is open and closed and hence $E_{2}^{\prime}=C E_{1}^{\prime} \cap c E_{2}$ is open and closed. Then $C_{E_{2}^{\prime}}$ is continuous. In general if

$$
E_{n}=\{x \mid F(x)>1-n \epsilon, n \leqq N\},
$$

then

$$
E_{n}^{\prime}=\left(C \bigcup_{i=1}^{n-1} E_{i}^{\prime}\right) \cap\left(c E_{n}\right)
$$

is open and closed and has a continuous characteristic function $C_{E_{n}^{\prime}}$. Also $\bigcup_{i=1}^{N} E_{i}^{\prime}=H$ and $E_{i}^{\prime} \cap E_{j}^{\prime}=0$ when $i \neq j$. It is easily verified that $\sum_{i=1}^{N} m_{i} C_{E_{i}^{\prime}}$ are extreme points of $S$ when $m_{i}= \pm 1$.

Let $k_{n}=n \epsilon / 2$ for $n<N$ and $k_{N}=1$. Form the functions

$$
\begin{aligned}
& g_{1}=k_{1}\left(-C_{E_{1}^{\prime}}+\sum_{j=2}^{N} C_{E_{j}^{\prime}}\right)+\left(1-k_{1}\right)\left(C_{E_{1}^{\prime}}+\sum_{j=2}^{N} C_{E_{i}^{\prime}}\right) \\
& =(1-\epsilon) C_{E_{1}^{\prime}}+\sum_{j=2}^{N} C_{E_{j}^{\prime}} \\
& g_{n}=\sum_{i=1}^{n}(1-i \epsilon) C_{E_{i}^{\prime}}+\sum_{j=n+1}^{N} C_{E_{j}^{\prime}} \quad \text { for } n<N \text {, } \\
& g_{N}=\sum_{i=1}^{N-1}(1-i \epsilon) C_{E_{i}^{\prime}}-C_{E_{N}^{\prime}}
\end{aligned}
$$

An element $g$ is in $K$ if and only if there exist an integer $n$, elements $g_{1}^{\prime}, g_{2}^{\prime}, \cdots, g_{n}^{\prime}$ in $K$, and positive numbers $a_{1}, a_{2}, \cdots, a_{n}$ with $\sum_{i=1}^{n} a_{i}=1$ such that $g=\sum_{i=1}^{n} a_{i} g_{i}^{\prime}[6$, Theorem 2.9, Chap. 2].

In the above set of equations each $k_{n}$ is positive or zero and is less than or equal to 1 . Hence $0 \leqq k_{n}, 1-k_{n} \leqq 1$ for $n=1, \cdots, N . k_{n}+\left(1-k_{n}\right)=1$. $g_{n}=k_{n} \bar{g}_{n-1}+\left(1-k_{n}\right) g_{n-1}, n=2,3, \cdots, N$ where $\bar{g}_{n-1}$ is equal to $g_{n-1}$ with the sign of $C_{E_{n}^{\prime}}$ changed. $\bar{g}_{n-1} \in K$ when $g_{n-1} \in K$; so by the theorem just quoted $g_{n} \in K$ when $g_{n-1} \in K$. Then since $g_{1} \in K$, all the $g_{n}$ are in $K$; in particular $g_{N}$ is in $K$.

For any $x$ in $H$ there is an $i \leqq N$ such that $E_{i}^{\prime}$ contains $x$. Then $\mid F(x)$ $-g_{N}(x) \mid \leqq \epsilon$. Hence $\left\|F-g_{N}\right\| \leqq \epsilon$; that is, for any $F$ in $S$ and $\epsilon>0$ there is an element $g$ in $K$ within $\epsilon$ of $F$. 
If we now choose a sequence $\{\epsilon(n)\}$ of $\epsilon$ 's, such that $\epsilon(n)=1 / n$, we can find a sequence of functions $g_{\epsilon(n)}$ in $K$ such that $\lim _{n \rightarrow \infty} g_{\epsilon(n)}=F$. Hence $F$ is in the closure of $K$; that is, $S$ is the closed convex hull of its extreme points.

6.5 CoRollary. If the normed linear space $X$ has property $\mathrm{P}_{1}$ and if the unit sphere $C$ of $X$ has an extreme point, then $C$ is the closed convex hull of its extreme points.

Proof. $X$ is equivalent to $C(H)$, the space of all real-valued continuous functions over some compact Hausdorff space $H$, by Lemma 5.4. Then $C(H)$ has property $\mathrm{P}_{1}$ and hence $H$ is extremally disconnected. Theorem 6.4 tells us the unit sphere of $C(H)$ is the closed convex hull of its extreme points. Then $C$ is the closed convex hull of its extreme points since an equivalence preserves extreme points, line segments, and distances.

6.6 CoRollary. If the normed linear space $X$ has property $\mathrm{P}_{1}$, if $X$ is infinite-dimensional, and if the unit sphere $C$ of $X$ has an extreme point, then $C$ has an infinite number of extreme points.

Proof. If $C$ has a finite number of extreme points, it is not the closed convex hull of its extreme points. Hence it does not have property $P_{1}$ by the corollary above.

Our next theorem gives a necessary and sufficient condition for a normed linear space $X$ to be equivalent to a space of type $B_{1}^{+}$which has a unit element. A space of type $B_{1}^{+}$which has a unit element is in turn equivalent to an abstract $(M)$-space $C(H)$.

6.7 Theorem. A normed linear space $X$ which has property $\mathrm{P}_{1}$ is equivalent to a space $Y$ of type $B_{1}^{+}$which has a unit element if and only if the unit sphere $C$ of $X$ has an extreme point.

Proof. Suppose the unit sphere $C$ of $X$ has an extreme point $u$. Let $x_{1} \geqq x_{2}$ mean $x_{1}-x_{2} \in C_{u}$. Then $X$ is equivalent to a space $C(H)$ of all continuous real-valued functions on some compact Hausdorff space $H$, by Lemma 5.4. The element $e$ which is identically equal to one on $H$ is the unit element in $C(H)$. Since $C(H)$ has property $\mathrm{P}_{1}$, every set in $C(H)$ which is bounded above has a least upper bound, by a previous lemma. Hence $C(H)$ is a space of type $B_{1}^{+}$and has a unit element $e$.

Suppose $X$ is equivalent to a space $Y$ of type $B_{1}^{+}$which has a unit element $e$. $e$ is an extreme point. Let $U$ be the equivalence between $X$ and $Y$ and consider the point $u=U^{-1}(e)$ in $X .\|u\|=1$ so $u$ is in $C$. Suppose $u=\left(x_{1}+x_{2}\right) / 2$ where $x_{1}$ and $x_{2}$ are in $C$.

$$
U(u)=e=\frac{U\left(x_{1}\right)+U\left(x_{2}\right)}{2}=\frac{y_{1}+y_{2}}{2}
$$

where $y_{1}$ and $y_{2}$ are in $S$ the unit sphere of $Y$. Then $y_{1}=y_{2}=e$ since $e$ is an 
extreme point. Hence $x_{1}=x_{2}=u$ and $u$ is an extreme point.

6.8 Theorem. Let $X$ be a normed linear space which has property $\mathrm{P}_{1}$. Then $X$ is reflexive if and only if it is finite-dimensional.

Proof. Let $X$ be reflexive and define $x \geqq y$ to mean $x-y \in C_{u}$. Then by Lemma 5.4, $X$ is equivalent to the space $C(H)$ of all real-valued continuous functions over some compact Hausdorff space $H$. Since a space is reflexive if and only if every space isomorphic to it is reflexive, $C(H)$ must be reflexive. $C(H)$ is an abstract $(M)$-space. But an abstract $(M)$-space is reflexive only when it is finite-dimensional [10, p. 1022]. Hence $C(H)$ is finite-dimensional and it follows that $X$ is finite-dimensional.

A theorem of Myers' [17, p. 404] gives us a bit of additional information about $H$. Since $H$ is a compact Hausdorff space, it is completely regular. Then Myers' theorem tells us that $C(H)$ is reflexive if and only if $H$ consists of a finite number of points.

6.9 TheOREM. The conjugate space $(A L)^{*}$ of an abstract $(L)$-space $(A L)$ has property $\mathrm{P}_{1}$.

Proof. $(A L)^{*}$ is an abstract $(M)$-space with a unit element $[10, \mathrm{p} .1021]$ and consequently is a space of type $B_{1}$ whose unit sphere has a least upper bound of norm one. Hence it suffices to prove that if a set $E$ in $(A L)^{*}$ is bounded above by an element $g_{0}$ in $(A L)^{*}$, then $E$ has a least upper bound, for then $(A L)^{*}$ has property $\mathrm{P}_{1}$ by Akilov's theorem.

Since $f \geqq g$ in $(A L)^{*}$ means $f(x) \geqq g(x)$ for all $x$ in the positive half-cone $K$ of $(A L)$, let $G=\left\{g \mid g_{0} \geqq g \geqq E, g\right.$ in $\left.(A L)^{*}\right\}$. Then $G \subseteq\left\{g \mid g_{0} \geqq g \geqq f_{0}\right\}$ for $f_{0}$ fixed in $E$. Hence $G$ is contained in a sphere in $(A L)^{*}$. If $K^{*}=\{f \mid f(x) \geqq 0$ for $x$ in $K\}$, then $G=\left[\bigcap_{f \in E}\left(K^{*}+f\right)\right] \cap\left(g_{0}-K^{*}\right)$. Since $K^{*}$ is weak star closed, so is $G$. Then $G$ is weak star closed and bounded and hence is weak star compact. If $x$ is in $K$ and $\phi(x)=\operatorname{glb}_{g} \in G g(x)$, then by compactness $G_{x}=G \cap\{g \mid g(x)$ $=\phi(x)\}$ cannot be empty. If $x_{1}, x_{2}, \cdots, x_{n}$ are in $K$, let $g_{1}, g_{2}, \cdots, g_{n}$ be such that $g_{i} \in G_{x_{i}}$ and define $g=g_{1} \wedge g_{2} \wedge \cdots \wedge g_{n}$. Then $g_{i}\left(x_{j}\right) \geqq \phi\left(x_{j}\right)$ for all $i, j$ since $g_{i} \in G$, so $g\left(x_{j}\right) \geqq \phi\left(x_{j}\right)$ for all $j$. Also $g\left(x_{j}\right) \leqq g_{j}\left(x_{j}\right)=\phi\left(x_{j}\right)$ for all $j$ so $g\left(x_{j}\right)=\phi\left(x_{j}\right)$ and $g \in \bigcap_{i=1}^{n} G_{x_{i}}$. Then by compactness $\bigcap_{x \in K} G_{x} \neq 0$.

If $g^{\prime}$ and $g^{\prime \prime}$ are in $\bigcap_{x \in K} G_{x}$, then $g^{\prime} \wedge g^{\prime \prime}$ is in $G$. $\left(g^{\prime}-\left(g^{\prime} \wedge g^{\prime \prime}\right)\right)(x) \geqq 0$ for all $x$ in $K$ and if $g^{\prime}>g^{\prime} \wedge g^{\prime \prime}$ there is an element $x_{0}$ in $K$ such that $\left(g^{\prime}-\left(g^{\prime} \wedge g^{\prime \prime}\right)\right)\left(x_{0}\right)$ $>0$; that is, $g^{\prime}\left(x_{0}\right)>\left(g^{\prime} \wedge g^{\prime \prime}\right)\left(x_{0}\right)$. But this is impossible since $g^{\prime}$ is in $G_{x_{0}}$. Hence $g^{\prime}=g^{\prime} \wedge g^{\prime \prime}$. Likewise $g^{\prime \prime}=g^{\prime} \wedge g^{\prime \prime}$ so $g_{1}=g_{2}$ and the intersection contains only one element, which is a least upper bound of $E$.

6.10 Corollary. The second conjugate space $(A M)^{* *}$ of an abstract $(M)$ space $(A M)$ has property $\mathrm{P}_{1}$.

Proof. $(A M)^{*}$ is an abstract $(L)$-space $(A L)[10$, p. 1021], and hence $(A M)^{* *}=(A L)^{*}$ has property $\mathrm{P}_{1}$. 
6.11 Corollary. An abstract $(M)$-space $(A M)$ has property $\mathrm{P}_{1}$ if and only if there is a projection $T,\|T\|=1$, of $(A M)^{* *}$ onto $Q(A M)$, where $Q$ is the natural mapping of $(A M)$ into $(A M)^{* *}$.

Proof. If there is a projection $T,\|T\|=1$, of $(A M)^{* *}$ onto $(A M)$, then $(A M)$ has property $\mathrm{P}_{1}$ by Theorem 3.2.

If there is no projection of norm one of $(A M)^{* *}$ onto $Q(A M)$, then $Q(A M)$, and hence $(A M)$, does not have property $\mathrm{P}_{1}$.

The real line $R$ is the simplest example of a space which has property $\mathrm{P}_{1}$. That it has this property was first shown by applying the Hahn-Banach theorem [3, p. 55] to the identity transformation of $R$ onto $R$, since by the theorem there is an extension of any linear functional on $R$ to a normed linear space containing $R$. It is easily seen that $R$ satisfies the conditions of Akilov's theorem. A result of Phillips' $[18$, p. 358] showed that $(m)$, the space of all bounded sequences, has property $\mathrm{P}_{1}$. It is easily verified that $(m)$ also satisfies the conditions of Akilov's theorem. Finally, the space $M$ of all essentially bounded measurable functions on the unit interval satisfies the conditions of Akilov's theorem and hence has property $P_{1}$.

Each of the known examples of spaces having property $P_{1}$ is the conjugate space of an abstract $(L)$-space. Hence each of the examples has a concrete representation as the space of all continuous real-valued functions over some extremally disconnected compact Hausdorff space $H$. It is an open question as to whether or not a necessary condition for a space $X$ to have property $\mathrm{P}_{1}$ is that $X$ is equivalent to the conjugate space of an abstract $(L)$-space.

\section{BIBLIOGRAPHY}

1. G. P. Akilov, Necessary conditions for the extension of linear operations, C. R. (Doklady) Acad. Sci. URSS. N. S. vol. 59 (1948) pp. 417-418. (Russian)

2. - On the extension of linear operations, C. R. (Doklady) Acad. Sci. URSS. N. S. vol. 57 (1947) pp. 643-646. (Russian)

3. S. Banach, Théorie des opérations linéaires, Warsaw, 1932.

4. J. A. Clarkson, $A$ characterization of C-spaces, Ann. of Math. vol. 48 (1947) pp. 845-850.

5. M. M. Day, $A$ review of Akilov's "On the extension of linear operations," Mathematical Reviews vol. 9 (1948) p. 241.

6. - Lecture notes for theory of linear operations, University of Illinois, 1947, unpublished.

7. G. Fichtenholtz, and L. Kantorovitch, Sur les opérations linéaires dans l'espace des functions bornés, Studia Mathematica vol. 5 (1934) pp. 69-98.

8. E. Hewitt, A problem of set-theoretic topology, Duke Math. J. vol. 10 (1943) pp. 309-333.

9. S. Kakutani, Concrete representation of abstract $(L)$-spaces and the mean ergodic theorem, Ann. of Math. (2) vol. 42 (1941) pp. 523-537.

10. - Concrete representation of abstract (M)-spaces, Ann. of Math. (2) vol. 42 (1941) pp. 994-1024.

11. L. Kantorovitch, Linear operations in semi-ordered spaces, I, Rec. Math. (Mat. Sbornik) N. S. vol. 7 (1940) pp. 210-279.

12. - Lineare halbgeordnete Räume, Rec. Math. (Mat. Sbornik) N. S. vol. 2 (1937) pp. 121-168. 
13. - Sur la théorie generale des opérations dans les espaces semi-ordonnés, C. R. (Doklady) Acad. Sci. URSS. N. S. vol. 1 (1936) pp. 283-286.

14. S. Mazur, Über konvexe Mengen in linearen normierten Räumen, Studia Mathematica vol. 4 (1933) pp. 70-84.

15. E. J. McShane, Integration, Princeton University Press, 1944.

16. F. J. Murray, On complementary manifolds and projections in spaces $L_{p}$ and $l_{p}$, Trans. Amer. Math. Soc. vol. 41 (1937) pp. 138-152.

17. S. B. Myers, Spaces of continuous functions, Bull. Amer. Math. Soc. vol. 55 (1949) pp. $402-407$.

18. R. A. Phillips, On linear transformations, Trans. Amer. Math. Soc. vol. 48 (1940) pp. $516-541$.

19. A. Sobczyk, Projection of the space $(m)$ on its subspace $\left(c_{0}\right)$, Bull. Amer. Math. Soc. vol. 47 (1941) pp. 938-947.

20. M. H. Stone, Boundedness properties in function lattices, Canadian Journal of Mathematics vol. 1 (1949) pp. 176-186.

UNIVERSITY OF ILLINOIS, URBANA, ILL.

Florida State University, Tallahassee, Fla. 